\title{
Condición juvenil contemporánea: reflexiones frente a las realidades del actual contexto sociohistórico y laboral
}

\author{
María Eugenia Pico Merchán \\ Uni*versidad de Caldas, Manizales, Colombia. \\ Email: maria.pico@ucaldas.edu.co \\ José Hoover Vanegas García \\ Universidad Autónoma de Manizales, Manizales, Colombia. \\ EMail: hovg@autonoma.edu.co
}

\begin{abstract}
Resumen: ${ }^{1}$ El artículo aborda la propuesta temática de las nuevas realidades del contexto socio histórico y, en especial, el laboral, y su relación con la condición juvenil contemporánea, entendida ésta como noción que instituye un referente desde el cual se puede entender la multiplicidad de formas de ser joven en los diferentes contextos. En un primer momento se desarrolla el recorrido de la noción de condición juvenil, a la luz de los cambios y desplazamientos teóricos de ésta, según las diferentes tendencias que la han reconceptualizado, y en un segundo momento se pretende dar cuenta de cómo el contexto laboral, desde sus diferentes formas adoptadas en la modernidad tardía, configuran la construcción de las identificaciones de los jóvenes en el ámbito laboral como espacio social de relaciones.
\end{abstract}

Palabras clave: condición juvenil, desplazamientos, contexto sociohistórico, contexto laboral.

\section{Youth's contemporary condition: an analysis in regards the realities of the present social, historic and labor context}

\footnotetext{
Abstract: The article discusses the proposal of the new realities of social and historical context and, in particular, the labor context, and its relation to youth's contemporary condition, understood as a concept that establishes a reference from which we can understand the multiplicity of ways of being young in the different contexts. The first part develops the route of the notion of youth condition, in the light of its theoretical displacements, according to the different trends that have reconceptualized it, in a second stage, it gives account of how the employment context, from its different forms it has taken in late modernity, build up the identifications of young people in the workplace as social space for relationships. labor context.

Key words: condition of youth, desplacements, socio-historical context,
} 


\title{
Condição juvenil contemporânea: reflexões frente às realidades do atual contexto sócio histórico e do trabalho
}

\begin{abstract}
Resumo: O artigo aborda a proposta temática para as novas realidades do contexto social e histórico e, principalmente, do trabalho e sua relação com a condição juvenil contemporânea, entendida como um conceito que estabelece um ponto de referência a partir do qual se pode compreender a multiplicidade de formas de ser jovem em diferentes contextos. Inicialmente se desenvolve o percurso da noção de situação da juventude à luz das mudanças e deslocamentos teóricos desta, segundo as diferentes tendências que o reconceitualizaram. Num segundo momento procura explicar como os contextos do emprego em suas diferentes formas adoptadas na modernidade tardia configuram a construção das identificações dos jovens no âmbito do trabalho como espaço social de relações.
\end{abstract}

Palavras-chave: condição juvenil, deslocamentos, contexto sócio-histórico, contexto do trabalho.

\section{La condición juvenil y sus múltiples aristas}

Aunque todavía no existe un acuerdo entre los autores acerca de cuándo se dio la construcción y legitimación del concepto juventud, sí existen ya apuestas teóricas muy sólidas que dejan entrever momentos, acontecimientos y vertientes que empezaron a visualizar y justificar una etapa en la vida de los seres humanos que caracterizaba la manera de vivir y visionar el mundo de aquellas personas que no se podían ubicar ni como niños, ni como adultos (Pérez Islas 2008:9).

En las siguientes líneas se presentan los desplazamientos teóricos de la juventud en la medida que se da una apropiación del conocimiento sobre los jóvenes en diferentes momentos históricos y la distinción entre los poderes en disputa por parte de las disciplinas frente a dicho conocimiento sobre los y las jóvenes. Desde esta perspectiva, se establece en este articulo la postura o punto de vista relacional e histórico, que ha permitido según Castellanos (2011), la configuración de la juventud permeada por la producción de sujetos que han sido clasificados de diferentes maneras (biológica, demográfica, psicológica y desde las políticas) no ya referido concretamente a la producción de los jóvenes, sino a la producción de sujetos en condición juvenil.

Así se ha concebido la juventud bajo las posturas de: 1) La juventud desde su concepción psicobiologica. Se trata de la visión que apunta a los cambios psicológicos, psíquicos y biológicos que se suceden en el desarrollo de la persona, de forma cíclica, progresiva, en lo que se define como adolescencia”-acepción que significa que "adolece” de las formas estructuradas y procesos propios que ya han alcanzado los adultos- perspectiva influenciada por la psicología del desarrollo, el psicoanálisis y los estudios sociológicos de enfoque funcionalista, a la vez que involucra las características “normales” y “anormales” en el comportamiento de una per- 
sona joven o adolescente, vista la juventud como una etapa de "riesgo" o “peligro”(Bonder 1999, citado por Alpizar y Bernal 2003:105-123). 2) La juventud vista como dato sociodemográfico. Esta mirada se desarrolló en la segunda mitad del siglo XX, en la que se ubicó a la juventud como grupo de edad para efectos de estudio y análisis desde lo poblacional, visto como grupo homogéneo, sujeto de las estadísticas, de manera marcada integrado por todas las personas que coinciden en un grupo de edad definido por cortes resultantes de intereses de carácter de medición para control poblacional, de la inserción productiva o aun por formas de caracterización de los comportamientos de los jóvenes de manera general, con lo que se invisiblizan la diversidad y heterogeneidad en cuanto a condiciones y realidad de éstos. 3) La juventud desde la perspectiva de la moratoria social. Se relaciona con "un periodo de permisividad que media entre la madurez biológica y la madurez social” (Margullis y Urresti 1998: 2), es decir, tiene que ver con el tiempo intermedio en el que los jóvenes, principalmente los de las clases privilegiadas y las medias, retrasan los compromiso de matrimonio, tener hijos y que pueden dedicar mayor tiempo para el estudio y la capacitación; por esto también se conoce como concepción pedagógica de la juventud, porque ésta se define como fase para la formación y de moratoria social o de estadio de la vida de espera o postergación de responsabilidades económicas sociales y familiares. Los de los sectores populares no tendrían condiciones para acceder a la moratoria social, porque éstos deben ingresar tempranamente al trabajo, generalmente de baja remuneración y estatus, quienes también asumen tempranamente obligaciones familiares (formar familia con hijos), incluso durante la adolescencia, como es el caso de las mujeres de estos sectores. Esta perspectiva al enfatizar en que sólo podrían ser considerados jóvenes los sujetos de los sectores privilegiados, "los otros carecerían de juventud" (Margullis y Urresti 1998: 4), introduce un factor de exclusión y diferenciación social. 4) La juventud como agente de cambio y problema del desarrollo, “objeto” de políticas sociales. Se consideran dos vertientes: una que va desde la influencia del materialismo histórico, que basada en la visión idealista de la juventud, la concibe como “agentes” y motores de la revolución reconocido su aporte en procesos de cambio social significativos (el Mayo francés, la revolución cubana, los movimientos pacifistas). Según Alpizar y Bernal (2003:105-123) se tiende hacia una postura de naturaleza positiva, que asigna a la juventud como depositaria de las esperanza frente a los vientos de cambio de la realidad social. La otra vertiente se relaciona con problemas del desarrollo, debido a la presencia de condiciones sociales tales como el desempleo, el consumo de sustancias ilícitas, el embarazo en adolescentes, es decir, se identifica la población joven en riesgo o como grupo "vulnerable", cuya integración es necesaria para el desarrollo socioeconómico, a lo cual se proponen políticas sociales dirigidas a esta población. 5) La juventud como construcción sociocultural desde los estudios de las ciencias sociales. Esta visón es más reciente e incluye diversas perspectivas como la postura antropológica del culturalismo estadounidense de Margaret Mead, (1972/1928), quien analizó y comparó las vivencias marcadas por una transición suave y la educación impartida por la familia en las adolescentes nativas de la isla Tau, en Samoa, océano Pacífico, con la educación familiar y escolar, los conflic- 
tos, indecisiones e inestabilidades en esa misma etapa de la vida de las norteamericanas, en lo que encontró que eran muy disímiles, lo cual atribuyó a las diferencias entre culturas y los diferentes momentos de civilización que vivía cada sociedad. También Margaret Mead (1980/1972) publicó una serie de ensayos conceptuales en los que elaboró una tipología de sociedades de acuerdo con las interrelaciones entre las nuevas y las viejas generaciones, llamó sociedad posfigurativa a la colectividad que tiene una transformación lenta y en la que los jóvenes aprenden de los adultos, sociedad cofigurativa a la que tiene cambio moderado y en la que los jóvenes y los adultos aprenden de sus pares, y sociedad prefigurativa a la de cambio acelerado y en la que los adultos aprenden de los jóvenes (Pérez Islas 2008:13-14). Ruth Benedict (1938) abordó las diferencias entre la naturaleza y el comportamiento humano encontrando que existen una serie de mediaciones que parten de la cultura y que influyen en la conformación de contrastes y diferencias entre los individuos de acuerdo con los roles que desempeñan, tal es el caso de la condición del individuo como niño y como padre (joven/adulto), en la que se presentan las oposiciones responsabilidad-no responsabilidad, dominio-sumisión y sexualidad-asexualidad.

De la Escuela de Chicago fue Frederic Thrasher quien primero investigó sobre jóvenes. Su obra: The gang. A study of 1313 gangs in Chicago (1927), está dedicada a analizar las formas de integración de las pandillas juveniles, su estructura organizativa, las ocupaciones de sus miembros, las normas que las rigen, sus prácticas, fines y propósitos desde una perspectiva microsociológica, llegando a la conclusión que las bandas les proporcionan a los jóvenes lo que la exclusión social les niega, como la protección, el acompañamiento y la solidaridad. Por su parte, William Foote Whyte (1971/1943) estudió las condiciones de los jóvenes del vecindario de Corneville entre 1937-1940 distinguiendo a los habitantes de las esquinas por su desempleo y abandono escolar y los muchachos de colegio como los que tienen la posibilidad de educarse en niveles superiores y tener una vida de reconocimiento social y profesional. Whyte también describe y analiza las relaciones del líder con los integrantes del grupo y viceversa (Ibíd: 15).

A partir de los desarrollos de la sociología siguiendo la pista de Pérez Islas (2008), surge la propuesta de las generaciones, de José Ortega y Gasset quien expresa que una generación es un colectivo de hombres que coinciden en el mismo espacio y en el mismo tiempo, tienen la misma edad y poseen alguna relación vital; los periodos que caracterizan a cada generación pueden ser de dos tipos: épocas cumulativas, las que se caracterizan por la dirección de los ancianos y en las que los jóvenes se doblegan a ellos; y, las épocas eliminatorias y polémicas, en ellas son los jóvenes quienes llevan la dirección y orientación con su beligerancia constructiva. En La rebelión de las masas (1930), Ortega y Gasset respondiendo a la pregunta de quién manda en el mundo, dice que en el siglo XX son los jóvenes quienes mantienen la preeminencia, porque es la época en que el hombre es productivo, lo cual le permite crear su particular forma de vivir, de esta manera lo que está expresando es que cada generación tiene su propio 
estilo de vida, de lo cual se desprende que la edad no es una fecha concreta, sino una época que entrelaza diversas fechas, en la que se suelen realizar ciertas especificidades de ese ciclo de la vida.

Karl Mannheim en su trabajo El problema de las generaciones (1993/ 1928) cuestiona desde una teoría del relacionismo el planteamiento generacional de Comte por partir de un tiempo externalizable, mecanicista, lineal y mensurable, y la posición histórico-romántica de Dilthey, por considerar que el vínculo que unificaba los miembros de una generación no era del todo comprensible como comunidad de influjo espiritual y social. Para proyectar su planteamiento de lo que debe pensarse como una generación, Mannheim considera el tiempo como algo vivencial, al que se tiene acceso por un sinnúmero de apreciaciones, de acuerdo con el estrato generacional en el que se hallen los individuos; es decir, desde una situación social, lugar o estado específico que se ocupe en la sociedad, "en su sentido más amplio, se puede entender por situación de clase la afinidad de posición que están destinados a tener determinados individuos dentro de la contextura económica y de poder de su respectiva sociedad” (Mannheim 1993: 207), como quien dice, desde la posición social en la que se encuentren las personas en su colectividad, éstas tendrán unas vivencias y unos pensamientos determinados que les permite encuadrarse en un proceso histórico concreto; la conexión generacional se da por la adhesión que se presenta en los individuos al participar en sucesos y vivencias comunes vinculadas en el mismo momento histórico-social, lo que también coadyuda a establecer la unidad generacional; y sólo se puede decir que existe conexión generacional si los contenidos sociales reales y los contenidos espirituales tienen un vínculo efectivo para los sujetos que se hallan en la misma posición generacional.

Del enfoque funcionalista se puede mencionar a algunos autores, entre ellos Talcott Parsons, James Coleman y Shmel Eisenstadt. En este sentido, Parsons afrontó la temática de juventud al considerar el rol como interacción social de la persona con el sistema en el que vive; en dicho sistema cada individuo tiene una serie de roles que cumplir, los cuales se caracterizan por ser funcionales y contribuir a la integración del todo social; es decir, el rol es un papel que desempeña la persona como actor de un campo determinado del sistema social desde un interés particular de acción e interacción.

Entre los años setenta y ochenta, los ingleses del Centro de Estudios Culturales Contemporáneos - CCCS—, por sus siglas en inglés, de la Universidad de Birmingham aportan a la temática juvenil y en sus trabajos investigativos revelan los conflictos de las nacientes subculturas juveniles de clase media ocasionados por los cambios estructurales que se dieron en la Inglaterra de la posguerra; en ellos proyectan su mirada sobre el fenómeno que encarna la lucha de los jóvenes por el reconocimiento de sus subculturas por parte de las clases hegemónicas desde una perspectiva sociohistórica, en que las tensiones se libran desde espacios como los simbolismos, los estilos y las modas, y las formas de ser, que son el reflejo 
de las transformaciones y los cambios sociales de un nuevo sensorium juvenil que empieza a reconocerse como una nueva modernidad. Se destacan como autores a: (Hall, 1968; Jefferson, 1973; Clarke, 1973; Hebdige, 1974,1979; Willis, 1978), que analizaron a los subgrupos juveniles como contraculturas y formas de resistencia simbólica frente a las clases dominantes (Pérez Islas 2008:27-28).

\section{La emergencia de la noción de condición juvenil}

Ahora, la condición juvenil ha emergido como una categoría central en los estudios socioculturales de Iberoamérica y empieza a ser reconceptualizada, a partir de los desarrollos teóricos de diferentes científicos sociales (Feixa, 2011; Reguillo, 2010; Urteaga, 2009; Valenzuela, 2006; Pérez Islas, 2008; Aguilera, 2006; Muñoz, 2010, 2011); quienes han incursionado desde las experiencias de los y las jóvenes en los contextos situados de América Latina y de algunos países de habla hispana que han permitido su continua reflexión e indagación desde aquellas opacidades o cisuras que comúnmente han pasado desapercibidas. En este sentido es necesario ir perfilando que de acuerdo a Valenzuela "la juventud es un concepto vacío de contenido fuera de su contexto histórico y sociocultural” (2005: 19), en el que el autor enfatiza que lo juvenil no se puede analizar fuera de su contexto social y relacional, no sin antes dejar claro que no siempre desde lo juvenil se pueden dar respuestas abarcativas sobre el conjunto de elementos que dan cuenta de los proyectos de vida de los jóvenes, se precisa entonces de pensar la juventud "como construcción sociocultural históricamente definida” (Ibíd: 20).

Desde otra postura Reguillo argumenta sobre la inestabilidad y la incertidumbre en la que viven y habitan los jóvenes y es clara con respecto a la centralidad sociopolítica y capacidad analítica de la categoría condición juvenil a la que define "como el conjunto multidimensional de formas particulares, diferenciadas y culturalmente "acordadas” que otorgan, definen, marcan, establecen límites y parámetros a la experiencia subjetiva y social de los/las jóvenes [...] (Reguillo 2010:401). Continuando con la misma teórica, plantea dos entradas analíticas para el estudio de los jóvenes, en la medida de poder incursionar por un lado, con la dependencia de las estructuras sociales y por el otro, con base en la idea de que la condición juvenil no tiene sentido por sí sola, sino que se pretende integrar lo subjetivo como constitutivo del papel de los jóvenes como actores sociales, por esto en palabras de Reguillo [...] "la condición juvenil es un concepto que posibilita analizar, de un lado, el orden y los discursos prescriptivos a través de los cuales la sociedad define lo que es “ser joven” y, de otro, los dispositivos de apropiación o resistencia con que los jóvenes encaran estos discursos u órdenes sociales” (Ibíd: 402).

Por condición se entiende desde la visión de posiciones, categorías, clases, situaciones, prácticas, prescripciones, proscripciones que se asumen como esenciales y naturales al orden vigente, las cuales también tien- 
den a ser vistas como propias de estas edades, de aquí se desprende que la condición juvenil sea entendida como parte de los mecanismos tanto de carácter estructural como cultural que determinan los procesos de inserción de los jóvenes concretos, en una dinámica social, económica y cultural configurada.

Según Rossi, 2006, la condición juvenil desde la participación política, es interpretada en cierta medida como transitoria por algunos jóvenes, dicha participación confluye en el horizonte de estos jóvenes como medio, más no como parte constitutiva de su actoría, ni estructura la participación política de los mismos, además que ésta no representa un deber, ni fidelidad a las diferentes organizaciones en las que ellos participan, ya que la fidelidad se da en el sentido de los principios que las sustentan.

Margullis señala la significación social de la condición juvenil, en tanto es una noción no natural, ni esencial, que no depende de la asociación biológica que se inscribe en la reflexión de lo que sucede naturalmente. Desde esta mirada la noción de juventud no estaría circunscrita a la demarcación dada por la edad o el tiempo vivido por una persona, en razón a que la edad como criterio de orden biológico es desbordada y afectada por la complejidad de significaciones sociales y una de estas significaciones es la juventud que alude a una condición social (Margullis 2001: 41).

En este sentido, la condición juvenil no puede ser definida por un rango etario, en razón a su insuficiencia explicativa y abarcativa, puesto que no considera el contexto histórico y sociocultural de relaciones sociales, además de las particularidades específicas. Es por lo anterior, que hay que tener en cuenta como puntos clave para pensar la condición juvenil contemporánea, a los componentes de lo relacional y la identificación, además de su carácter de construcción sociocultural históricamente definida.

Con respecto a lo relacional se plantea que la concepción de condición juvenil es necesario incluirla dentro del sistema de relaciones sociales, de interacciones, de fuerza, de sentido que es posible definir según las características específicas y de situación espacio- temporal de los jóvenes donde de paso adquiere sentido (Castellanos, 2011), por tanto, lo relacional como elemento constitutivo de la condición juvenil se inscribe dentro de un entramado de relaciones y de naturaleza contextual a la vez que le imprime su carácter de sentido.

Para hablar de la identificación es pertinente referirnos a la perspectiva constructivista y procesual de Hall o teoría cultural inglesa (2003:16), la aborda como "proceso que actúa a través de la diferencia, entraña un trabajo discursivo, la marcación y ratificación de límites simbólicos, [...] Necesita lo que queda afuera, su exterior constitutivo, para consolidar el proceso”. Con esto se argumenta que se construye a través de la diferencia y que la identidad siempre es una relación con el otro; identidad y alteridad están en una relación dialéctica, en tanto obedece a la lógica del más de uno (el sujeto y ese “otro” con el que se identifica) con lo que implica la continua 
marcación y ratificación de limites simbólicos, la producción de efectos de frontera, es decir, necesita de lo que queda afuera (Derrida, 1891, Laclau, 1990; Butler,1993 citados por Hall 2003:18).

Así para Hall lo relacional es un elemento fundante en la constitución de la identidad teniendo en cuenta que las representaciones motivan la adhesión identitaria de los sujetos y lo hacen mediante la identificación, ésta siempre está en proceso, es siempre inestable, una fantasía de incorporación, por lo tanto, la identificación es un proceso de articulación, una sutura; así Hall (2003:20) plantea que la identidad es el punto de sutura entre, por un lado, los discursos que nos interpelan y tratan de "ponernos en nuestro lugar”, y por otro lado, los procesos y prácticas con que se construyen sujetos susceptibles de decirse.

Por tanto, para pensar la condición juvenil es pertinente analizar la identificación a la luz de una perspectiva alejada de la postura esencialista y como algo dado y fijo, mediante el reconocimiento de la expresión cambiante, dinámica, transitoria, fragmentada, flexible y contradictoria de las identidades particularmente las juveniles en un determinado contexto sociohistórico; entendida la condición juvenil como noción que instituye un referente desde el cual se puede entender la multiplicidad de formas de ser joven en los diferentes contextos, además de su carácter relacional como parte de las expresiones y representaciones que crean y recrean los actores juveniles y grupos sociales, que sólo se explican y definen en relación con los demás: en lo que compartimos, en las similitudes y en las diferencias, en la que las características de lo juvenil adquieren significación. "No existe un único modo de ser joven, no podemos hablar de juventud en singular. De acuerdo con el lugar que se ocupe en el espacio social, de acuerdo con el género, con los capitales materiales y simbólicos por los cuales se esté atravesado, se es joven de distinta manera” (Muñoz 2011:61).

\section{Globalización y su implicación en las condiciones laborales de los jóvenes}

A la par con el desmantelamiento de la matriz sociopolítica que daba soporte a la sociedad en conexión con el Estado representado en el movimiento obrero, aunque este fenómeno se presentó de manera diferenciada, se puede decir que en América Latina y en algunos países africanos se dio este proceso junto con los regímenes militares y las reformas al Estado de Bienestar y la consiguiente radicalización de las políticas neoliberales, concomitantemente se produjo en Europa un retroceso de las capacidades del Estado para articular a los sectores que se caracterizan por fuertes lazos de solidaridad (Rosanvallon 1995: 10 citado por Rossi 2006: 9); fenómeno de transformación llevado de la mano por la globalización. Idea que ha sido debatida por autores como Sen (2002), quien argumenta que el proceso de globalización no es una propuesta iniciada en la década de los 80, sino que ha sido un acontecimiento sistemático y continuo tan antiguo de enormes 
implicaciones en la vida social, cultural, política, económica y artística de la humanidad, no solamente de carácter económico como continuador del pensamiento ilustrado y burgués de inicios del siglo XX (Sen 2002).

Para autores como Bauman (2007:18) desde el ámbito de lo éticopolítico, la globalización es un fenómeno más profundo de lo que aparece a la vista, al dejar ver sus raíces y consecuencias sociales, es decir, muestra las estructuras del poder y de la sociedad que la misma ha generado, así como las consecuencias humanas de este nuevo ordenamiento. La demanda de la aplicación de la ley y el orden entendida casi exclusivamente como la demanda de protección personal, resulta en un enmascaramiento de la falta de certeza, desprotección, inseguridad, miedo y ansiedad que provoca esta sociedad globalizada, sentimientos que fragmentan y menoscaban la misma subjetividad.

En concordancia con Bauman (2006:136), actualmente se plantea una tendencia general consistente en limitar las funciones políticas del Estado relacionada con el tratamiento excesivo de los problemas de la ley y el orden, con el fin de "crear condiciones que despierten la confianza de los inversores, en consecuencia se requiere un control más estricto del gasto público, una reducción de la carga impositiva, una reforma del sistema de protección social y “desmantelar las rigideces del mercado laboral”(Ibíd.). La dimensión global de las posibilidades de elección del inversionista, frente a los límites estrictamente locales de la posibilidad de elección del proveedor del trabajo y del que lo demanda, crea una asimetría que subyace, a su vez, a la dominación del inversionista en el terreno de lo global y la misma movilidad.

Desde otras perspectivas teóricas de la sociología en cabeza de (Beck, 1998), ante las acometidas de la nueva situación derivada de las inseguridades, plantea que se debe abrir el debate sobre la configuración política de lo que ocurre con los procesos de incertidumbre:

“[...] esto presupone una decisiva critica de la ideología neoliberal de la globalización, de su unidimensionalidad económica, de su pensamiento único lineal, de su autoritarismo político en relación al mercado mundial, que se impone apolíticamente y que actúa de manera altamente política. [...] El choque de la globalización, característico de la transición a la segunda modernidad, provoca paradojas y exigencias en términos políticos, porque todos los agentes y organizaciones, y esto en todos los ámbitos de la sociedad, han de confrontarse con la dinámica de la globalización que transforma los fundamentos” (Beck 1998: 241-242).

Es por esto que para este autor es primordial la postura de las respuestas políticas que pueden perfilarse ante la globalización y las relaciona con cooperación internacional, participación en el capital, reorientación de la política educativa, alianza para el trabajo ciudadano, empresarios públicos y trabajadores autónomos, pacto social frente a la exclusión, (Ibid:242), 
propuestas que si bien son loables, evocan de una u otra manera la permanencia y persistencia del sistema capitalista neoliberal hegemónico, que con todas sus contradicciones continua vigente.

A partir de las visiones con énfasis en lo económico, los cambios derivados de los procesos de globalización como lo expresa Stiglitz (2006:287), supuestamente han reportado enormes beneficios a todos, sin embargo, ha sido confrontada en el mundo desarrollado y por los países en vías de desarrollo; por un lado, Estados Unidos y Europa ven en ella una amenaza de externalización empresarial en la que las industrias contratan y realizan actividades en países donde la fuerza de trabajo es de menor costo, por el otro lado, los países en vías de desarrollo han visto con temor las imposiciones economicistas de los países ricos frente a la deuda que han contraído con éstos, Igualmente ambos mundos observan que los intereses privados priman a costa de otros valores, con el consiguiente aumento de las desigualdades entre países, especialmente en materia de empleo, así Stiglitz afirma:

“...para gran parte del mundo, la globalización, al menos tal y como se ha gestionado, es como un pacto con el diablo. Unos pocos se hacen más ricos... la mayor integración en la economía global ha traído mayor inestabilidad e inseguridad, y más desigualdades. Y ha puesto en peligro los valores fundamentales” (Stiglitz 2006: 366).

Desde las implicaciones en la cultura señala García Canclini (1989:179), es posible plantear la doble agenda de la globalización para explicar las condiciones objetivas que permiten la distribución de los bienes y mensajes entre unos países más que en otros: por una parte, está el discurso que narra la expansión del capitalismo postindustrial y de las comunicaciones masivas como un proceso unificador/o articulación de empresas productivas, sistemas financieros, regímenes de información y entretenimiento que se convierten en oligopolios (Wall Street, MTV, CNN), con sus redes de consumidores transnacionales. Pero esta unificación de mercados materiales y simbólicos produce desigualdades y nuevas fronteras, además de las inequitativa distribución de dichos productos. Por otra parte, la agenda de la globalización se relaciona con las estrategias globales de las multinacionales y de algunos Estados que configuran máquinas segregantes y dispersadoras (Ibíd: 180). Así se plantean políticas de flexibilización laboral que llevan a desestimular la sindicalización de trabajadores, migraciones, mercados informales, externalización o realización de actividades de una empresa con trabajadores contratados en el extranjero, en este sentido se dice que la eliminación de trabas a las inversiones extranjeras ha sido el principal recurso para eliminar normas de los sindicatos, del sector asistencial y ecológico con el cual los Estados modernos contenían la ambición de los capitales, por lo anterior, para este autor: "globalización no significa únicamente libre circulación de bienes y mensajes; también debe incluirse en su definición el poder de “exportar fuentes de trabajo” a donde sean más bajos los costos laborales y las cargas fiscales” (García Canclini 1989:180). 
Para la juventud es importante revelar según (Ortiz 1998: 63), la perspectiva del macroproyecto de consumo como una nueva forma de unidad, dependencia imaginaria y de enfoque económico. Este nuevo macroproyecto transnacional desde lo conceptual, como lo señala Ortiz:

"posibilita la idea de unidad en la desterritorialización como memoria colectiva masiva que genera una ética y modo de conducta compartida, tal es el caso de la promoción de estrellas e ídolos en los medios masivos de comunicación, que para los jóvenes es asimilable a compartir los mismos gustos, las mismas inclinaciones, que circulan en un espacio de expectativas comunes. Así se unifican gustos, pero también se les frustra, gracias a la variedad y cantidad no accesible a todos” (Ibíd: 37).

Siguiendo a Ortiz, plantea que a la vez que se unifican los deseos del consumo, se les separa socialmente, confirmando la no superación de las desigualdades económicas entre los distintos actores sociales. Esta ética del mercado y del consumo posee en su interior dos agendas contradictorias de lo global antes mencionadas, es decir, unifica y desintegra; homogeniza y segrega (Ibíd: 37).

\section{Cambios en el contexto de lo laboral desde lo sociohistórico y cultural}

Con el anterior antecedente de los procesos de globalización y contexto sociohistórico nos detendremos en el contexto del trabajo, toda vez que asistimos desde la década de los noventa a una transformación importante en las relaciones entre capital y el trabajo. Siguiendo a Weber, Durkheim y Marx, (1997), se plantea que es a través del trabajo que los sujetos adquieren una identidad socialmente reconocida o un anclaje que los vincula a lo social; para otros teóricos en la llamada sociedad en tiempos de la gran vinculación de acuerdo con Bauman (2001) y la sociedad salarial según Castel (2004), se presentó la coincidencia de tres formas dominantes de concreción de las relaciones de trabajo alrededor de la sociedad industrial con dominio del capitalismo, aunque sin un encadenamiento lineal: la condición proletaria que se asociaba con el proletario, cuyas condiciones salariales sólo le permitían la subsistencia y para su proceso de reproducción social, las relaciones con la empresa se caracterizaban por su marginalidad, precarización salarial y débil vínculo con la organización empresarial; la condición obrera con fuertes vínculos a la empresa, aseguraba derechos y prestaciones sociales fuera del trabajo como seguros contra (accidentes de trabajo, enfermedad profesional, jubilación) que permitían una participación en la vida social y proceso de reproducción social, tales como el consumo, vivienda, educación y ocio, "es la imagen de la integración en la subordinación” (Castel 1997:374); y la denominada condición salarial ampliada a las clases medias y de mayor estatus que de acuerdo con Castel posibilitó: 
“la profesionalización de puestos de trabajo, el desarrollo de segmentos profesionales alrededor del sector terciario; el salariado daba fundamento a una identidad social y estaba ligado al progreso económico y a la movilidad social ascendente” (Ibíd: 374).

Ahora la fase actual del capitalismo se expresa como una contradicción, según Bialakowsky (2009:52) referida al cambio de carácter del trabajo y a la consiguiente expansión de un proceso de exclusión social y laboral, en sentido opuesto, a la fase de consolidación del capitalismo que trajo consigo la expansión del trabajo y del consumo a la vez como principales estrategias de inclusión social, por tanto en la etapa actual se da una mutación de la relación capital-trabajo que ha alterado las condiciones y calidades del empleo, con abandono del pleno empleo. Para Bialakowsky et al (2003), las transformaciones se enmarcaron en lo que estos autores denominaron "un proceso de trabajo social" y en una estrategia de gubernamentalidad ${ }^{2}$ (Foucault, 1978), que se plasmaron en el nivel de cambio en las racionalidades políticas y de tecnologías de gobierno (Rose y Miller, 1992 citado por Bialakowsky et al 2009: 53).

Así en concordancia con los planteamientos de Castel, estos autores argumentan que los cambios se evidenciaron en una racionalidad política marcada por el keynesianismo (corriente de pensamiento económica fundamentada en la acción o intervención del gobierno para impulsar la actividad económica), que llevaron al empleo asalariado estable, remunerado, con capacidad de ahorro; los sistemas de protección social entendidos como extensión de la misma relación con el salario y por último, la unidad familiar como institución que fungía como mediadora en la provisión de bienes y servicios obtenidos vía salario y las prestaciones sociales; se alteraron en razón del tránsito hacia una racionalidad política de carácter neoliberal que introdujo cambios radicales en el trabajo en su base técnica y en las formas de contratación, a la vez nuevas formas de gestión de los riesgos basados en la estrategia de responsabilización individual (Bialakowsky et al, 2009:53), o lo que Castel plantea como la gran transformación producida por el advenimiento del capitalismo postindustrial y es la dinámica llamada descolectivización o reindividualización (Castel 2010: 24), dinámica representada en la organización del trabajo relacionada con la individualización progresiva de las tareas, que exige la movilidad, la adaptabilidad, la asunción de responsabilidades por parte de los operadores pues a decir de Castel ya el término “trabajador” ha dejado de tener actualidad. El concepto de colectivos de trabajo se desbordó hacia su disolución, en tanto con la predominancia del trabajo en red es notoria la conexión-desconexión -reconexión de las personas en vista de la incursión de nuevas formas extremas de trabajo inmaterial que se presentan bajo la modalidad de los proyectos y formas de vinculación laboral flexibles.

Ahora es preciso señalar que posterior a este proceso del salariado, en la década de los 90 se planteó un debate académico alrededor de la tesis de la crisis del trabajo o del fin de la centralidad del trabajo surgido en Europa y Estados Unidos con autores representativos como Rifkin, Gorz y 
Claus Offe. Consecuentes con lo anterior, Rifkin planteó el "fin del trabajo" (1996), elemento teórico de amplia critica en los sectores académicos de los estudios del trabajo sobre todo los latinoamericanos, en cuanto a que se trataba con esta postura de restarle centralidad al trabajo como eje de la vida social, económica y política, si bien como dice De la Garza esto se ha dado, sin embargo, es diciente la cantidad de fuerza de trabajo inserta en los procesos productivos en especial en grandes empresas y además obviamente con otros sistemas de contratación, flexibilidad, desempleo, ampliamente descritos por (Bauman, 2007; Castel, 2010). De acuerdo con Gorz, el fin del trabajo no es tan concluyente en la medida que lo que se estaría presentando es el fin del empleo asalariado, con la existencia de otras formas laborales por fuera de una relación salarial estable (Gorz, 1998). Claus Offe otro teórico del trabajo, enunció después de los años 80 y 90 el fin de la centralidad del trabajo visto como fundamental en la organización de las relaciones e identidades sociales. Offe asocia la crisis del trabajo con la evidente fragmentación de los mundos de vida de los trabajadores (trabajo, familia, tiempo libre, consumo, entre otros), a la par con las transformaciones en la estructura productiva, el auge del sector terciario y el cambio en la composición de las ocupaciones, marcado por la disminución de los obreros y el auge de los empleados de cuello blanco (Offe, 1996).

En el debate sobre el fin del trabajo, Castel (1997, 2010), argumenta que el trabajo no desaparece sino que se transforma, desarrollándose nuevas modalidades de trabajo, proceso en el que el trabajo estable se fragiliza en razón al desempleo, ser desempleado no significaría vagancia o falta de voluntad para trabajar, o tener las condiciones para trabajar, ya no garantiza una inserción en el mundo del trabajo, sino que lo anterior se avizora como problemática estructural de las sociedades posmodernas.

Las anteriores propuestas se complementan con la visión posmoderna caracterizada por la fragmentación social, en tanto la crisis de la sociedad actual es en todas las esferas de la acción entre las que se encuentra la del trabajo. Para los seguidores de esta perspectiva, es evidente que ya no serían posibles los proyectos globales y universalistas basados únicamente en la razón científica, puesto que incursionan otras miradas desde lo local y particular, además de la pérdida de relevancia de los metadiscursos y de los sujetos sociales, en especial de la capacidad de articulación social y política de la clase obrera, otrora en la industrialización vista como actor de referente social e identificación de la sociedad y sus instituciones (Carpio y otros: 2000). Paralelo a esto, se daría paso a la fragmentación de las nuevas subjetividades sociales, identidades, culturas y concretamente con las posibilidades de creación de proyectos que llevarían a transformaciones de la sociedad, con la consiguiente "atomización del mundo del trabajo, de los trabajadores, y de todos aquellos espacios donde irrumpía lo colectivo como potencial germen de cambios profundos en la sociedad" (Aravena 1999: 13). En últimas, estas posturas del fin del trabajo coinciden en plantear el fin del trabajo como articulador de identidades y de sensibilidades sociales y culturales. 
De acuerdo con De la Garza (2001), en cuanto a la posibilidad de construir subjetividades e identidades colectivas en torno al trabajo - posición que han disentido las perspectivas posmodernas y de fin del trabajodicho teórico nos presenta otra mirada que apuesta a reconocer nuevas formas de identidad en el trabajo; así señala que antes de proceder a negarlas, es necesario examinarlas a la luz de las transformaciones que se han presentado en el mundo del trabajo. Por esto ha propuesto una nueva articulación entre subjetividades, acción y las estructuras, en la que los sujetos no actúan sólo en función de su ubicación en las estructuras, sino que los sujetos llevan a cabo un proceso de reflexión, de dar sentido y decisión sobre sus cursos de acción y de vida. La subjetividad según de la Garza:

"no es una estructura que da sentido de uno a uno, sino un proceso que pone en juego estructuras subjetivas parciales (cognoscitivas, valorativas, de la personalidad, estéticas, sentimentales, discursivas y de formas de razonamiento); es subjetividad con estructuras parciales en diferentes niveles de abstracción y profundidad que se reconfigura para la situación y decisión concretas" (De la Garza 2000: 28-29).

En este sentido la subjetividad según de la Garza, es un proceso de dar sentido a situaciones específicas, que admite incoherencias, además de discontinuidades y contradicciones. La identidad la concibe de la Garza como una forma particular de subjetividad que se da en una diversidad de expresiones y manifestaciones:

"En tanto sentido de pertenencia colectiva, con sus signos compartidos, su memoria colectiva, sus mitos fundacionales, su lenguaje, su estilo de vida, sus modelos de comportamiento y, en niveles superiores, sus proyectos y enemigos comunes; esta identidad como la subjetividad, puede reconocer niveles desde los más ambiguos hasta los más específicos y, en esta medida, aceptar la pregunta. ¿Identidad para cuales espacios de acción?” (De la Garza 2000:29).

\section{La vinculación entre los jóvenes y el actual contexto laboral}

Para el caso de los jóvenes las posturas de De la Garza son esclarecedoras, en tanto, en este contexto socio histórico y económico se plantea que esta población es afectada por las mismas fuerzas del mercado, ya que frente al trabajo le restringen e inmovilizan las posibilidades de elección para acceder a puestos laborales, en contraposición a la libertad y flexibilidad del gran capital inversor para imponer las leyes y normas globales. Este fenómeno se observa en la mayoría de países Latinoamericanos e incluso los europeos como España, en donde el desempleo juvenil ronda por el 22\% uno de los más altos de dicho continente. Quizá esta sería una explicación para el proceso de movilización de los “indignados” en el 2012 que en su mayoría eran jóvenes desempleados que propugnaban por 
cambios en la sociedad española y en el sistema financiero global. Resulta paradójico que la juventud a pesar de manejar y consumir los nuevos medios de procesamiento de información y de una mayor participación de redes a distancia, e incluso más años de educación, no ve que se traduzca en mayor presencia en las instancias de decisión política, a la par de un mejoramiento de condiciones materiales, planteando desafíos a la inclusión (Hopenhayn, 2008, p. 18). En el mismo sentido, se argumenta que las reclamaciones de los jóvenes apuntan en asuntos relacionados con la inconformidad en cuanto a las condiciones precarias, los altos costos de vivienda, imposibilidad de acceder a una pensión y el mejoramiento de condiciones de la educación, son aspectos que sugieren que la situación de la condición juvenil en los países periféricos como Colombia es a todas luces también precaria.

Así la expresión de la globalización en Colombia que afecta lo local, encuentra su nicho en el sistema económico y las relaciones productivas que privilegian el modelo exógeno de afuera hacia adentro, basado en la competitividad global de los mercados, con un débil apoyo a la investigación, la innovación y el desarrollo de los sistemas productivos locales, afectando el capital social y el conocimiento producido. De ahí que sería deseable para el contexto local, el planteamiento de un sistema territorial de innovación de carácter endógeno, de adentro hacia afuera, que surja como parte de los procesos de desarrollo gestados en los territorios regionales, solidario y colaborativo, basado en el conocimiento, como principal factor de desarrollo de los sistemas productivos locales, se plantea como una necesidad (Acevedo, 2010).

Los estudios en las sociedades latinoamericanas (De la Garza, 2001, Neffa, 2001), han coincidido en asegurar que el trabajo no ha desaparecido según lo argumentado por Rifkin, pero si se ha transformado, es así que es necesario estudiarlo desde las condiciones en que se realiza y sus expresiones en la actualidad. Para Cortés (2000), el trabajo continúa siendo fundamental y relevante en la sociedad, como se aprecia en las diferentes transformaciones y dinámicas socio laborales surgidas en el trabajo asalariado y el creciente aumento del trabajo informal, con lo cual se evidencia que la experiencia laboral continua siendo esencial en la vida de las personas. Con lo anterior, es posible plantear que la tesis sobre la crisis del trabajo en la mayoría de países de América Latina, está estrechamente ligada a una crisis de precarización de las actividades, de exclusión social vinculada a la creciente pobreza, por tanto, no se puede enfatizar que en nuestro contexto latinoamericano el trabajo no es relevante, sino que los cambios han llevado a procesos de pérdida de las condiciones de seguridad y de realización que antes si se garantizaban como el trabajo protegido, con seguridad social integral, estabilidad laboral, por tiempo indefinido, entre otros.

Para teóricos como De la Garza, (2001) la crisis del trabajo en Latinoamérica adquiere otras connotaciones diferentes a los llamados países centrales, en razón a que es sobre todo la crisis del empleo en las unidades de tamaño mediano y pequeñas, además por la presencia de 
microunidades de trabajo que son tan pequeñas y a la vez separadas unas de otras, esto tanto en los sectores de servicios, manufacturero y de autoempleo; de lo anterior se desprende que la fragmentación de las identidades de los trabajadores "no es por la diversidad de mundos de vida (trabajo, familia, ocio, etc.) para la mayoría de la población en América Latina, sino por laborar en unidades tan pequeñas y desarticuladas. Sin embargo este sentido de la fragmentación tiene una parte importante de homogeneidad: la precarización” (De la Garza 2001:28). Continúa argumentando este autor que otra característica de los trabajadores de estas unidades de trabajo es que son de baja calificación y de condiciones precarias semejantes y, concluye que "tampoco se trata de un aumento espectacular del desempleo, sino un incremento sustancial de las actividades precarias” (Ibíd).

Por esto el capitalismo de marcado corte neoliberal, ha permeado todo el tejido social, en la que los jóvenes se encuentran inmersos; es indudable que los jóvenes actualmente desde el punto de vista de las nuevas realidades, se dedican a experimentar caminos diversos para sobrevivir e innovar, García Canclini explica el evidente cuestionamiento y malestar de los jóvenes manifestado en discursos de carácter cultural representado en canciones, videos, blogs, twitters y en prácticas que conllevan autoorganización o como muchos que deciden emigrar, ello ha promovido la emergencia y la importancia de la creatividad en muchos ámbitos vitales. Lo anterior se presenta, porque de la mano del avance de las tecnologías de la comunicación y la informática, la expansión de la conectividad ha posibilitado a las personas el desarrollo de la creatividad con el fin de alcanzar por medio de ésta mejores condiciones de vida y trabajo, en sentido contrario, las desventajas socioeconómicas y el riesgo de exclusión representado en inestabilidad y precariedad laboral, se vinculan a la falta de competencia en las nuevas tendencias de innovación y creatividad (García Canclini 2012: 20). Siguiendo a García Canclini argumenta que no basta con concebir la creatividad

“como recurso para superar estructuralmente la pérdida de seguridad en el trabajo, la inestabilidad y precarización. Lo que logramos captar son algunas vías a través de las cuales ciertos sectores se reubican. Una ponderación adecuada de su alcance requiere verlos en el contexto de creciente desempleo; en el marco de la tercerización y la subcontratación temporal, las redes informales donde la sobreexplotación del trabajo y del tiempo impide a muchos concluir la formación escolar y reciclarse “ (Ibíd).

Así mismo, la condición juvenil ha mutado en diferentes perspectivas (la cultural, la del consumo, la de la participación) pero especialmente ha desplegado su proceso de valorización como nueva fuerza de trabajo. Según (Pérez Islas, 2010), se da la confluencia de trabajos enmarcados en la incertidumbre en los que los jóvenes son importantes por el presentismo, en tanto no construyen proyectos de largo alcance, sino que los proyectos que construyen son marcados por la rapidez, lo efímero y la intensidad, es el caso típico de los jóvenes trabajadores del mundo del espectáculo, de las 
entidades financieras, corredores de bolsa, la industria de la moda, la publicidad y los deportes, entre otros. Una segunda forma de valorización de la fuerza de trabajo juvenil es la que involucra de acuerdo con Pérez Islas, (2010) la superexplotación que se relaciona con "la reducción del fondo de consumo de los trabajadores por la vía de la disminución salarial o de la masa salarial, dando contexto a la precarización del trabajo” (Ibid: 7). En esta forma de valorización laboral este autor menciona a las jóvenes de las maquilas, a los y las jóvenes de cadenas comerciales y de alimentos, los jóvenes de los callcenters, cuya característica común de dichas ocupaciones es que son "trabajadores de rutina" (Ibid:6-12).

Un tercer mecanismo de valorización de la fuerza de trabajo juvenil apunta hacia el desafío que va más allá de la incertidumbre, y es la que tiene ver como insumo al riesgo en tanto única forma de incrementar su valor, mediante estrategias de enfrentar peligros y de resistencia en la que se arriesga hasta su vida, son los jóvenes pertenecientes a las ventas ambulantes, las ventas de la calle, la comercialización legal o paralegal de zapatos, discos, ropa pirata, y las ventas de sustancias consideradas ilegales y hasta el tráfico de armas y personas. Estos tipos de trabajo se realizan por lo general de manera grupal, así se da la presencia de organizaciones de vendedores ambulantes, la formación de mafias de armas o drogas que se constituyen en redes de pertenencia que instrumentalizan a sus miembros mediante prácticas protectoras, sancionatorias y de autorización para dichas actividades. Estos tres horizontes de valorización arriba descritos, envuelven a los sujetos en condición juvenil en nuevos modos de ser en el mundo, lo que deviene en subjetividades más proclives a la inmediatez, al cambio, a vínculos débiles, a la incertidumbre, a la sensación de estar a la deriva y a la flexibilidad básicamente en el ámbito de lo laboral.

A lo anteriormente propuesto es necesario introducirle un aspecto de relevancia que también es central en la constitución de la condición juvenil y es el cuerpo juvenil, toda vez que la dimensión corporal es un componente de la biopolítica, como mecanismo de regulación y control de la sociedad, desde su interior (Piña Mendoza; 2004). Para autores como Reguillo, 2000 y Pérez Islas, 2010, la condición juvenil desde esta visión así planteada, permite entender una biopolítica del cuerpo-mercancía, concepto que engloba las estrategias laborales antes descritas en un entorno de valorización particular de lo juvenil, al igual que como mecanismo de obtención "de mayor plusvalía del "capital corporal” que tienen las nuevas generaciones” (Pérez Islas, 2010:12).

Por último, para pensar la relación condición juvenil y contexto laboral es imprescindible reflexionar sobre las articulaciones entre estructuras, subjetividades y las acciones en la agencia juvenil en razón a los cambios que se han dado, ya que sus referentes de reflexividad son distintos, su lógica es otra -su horizonte ya no es a largo plazo-, se asume el riesgo y la incertidumbre como condición para obtener mejores beneficios enmarcados en las reglas y recursos propios de las estructuras sociales, que cada vez son menos regularizadas puesto que no son ya el soporte o guía seguro de 
anclaje de los sujetos. Para De la Garza (1994:369), cuando se analizan las estructuras sociales, tener en cuenta que éstas se encuentran en diferentes niveles desde las de naturaleza económica, las políticas, las culturales pasando por las subjetivas donde se presentan incoherencias, discontinuidades, por tanto, una parte es dependiente del individuo, y otra de la sociedad que le está imponiendo sus reglas y recursos. Parafraseando a Castel, la condición juvenil constituye la categoría que mejor simboliza las rupturas subjetivas de las transformaciones en el mundo del trabajo y que más se ve afectado por la sensación de incertidumbre que sumerge a los sujetos, tras esta serie de quiebres y rupturas (Castel 2010:112).

\section{Conclusiones}

El resurgimiento de nuevas formas de plantear la incursión de los jóvenes en las sociedades, como lo es la interpretación de las experiencias que los atañe, ha implicado el reconocimiento de la diversidad, heterogeneidad, movilidad, lo efímero, lo circunstancial, lo indeterminado que acompaña muchas de las expresiones de lo juvenil. La mirada de la condición juvenil es una apuesta que en la contemporaneidad nos estimula a indagar en otras discursividades diferentes a la oficial/hegemónica, que apunte a la visibilidad de las prácticas que emergen en contextos específicos, como el laboral, donde los y las jóvenes configuran sensibilidades, utopías y transitoriedades, en tanto necesitan el reconocimiento en su especificidad y a la vez como parte de un todo en el sentido de unicidad. Junto a la erosión de algunos referentes como lo fue el trabajo e incluso la escuela, y frente a los contextos de violencia latinoamericanos, es importante plantear que lo que buscan los jóvenes y las jóvenes es su inclusión como pares, a la vez articulados en las redes institucionales y sociales donde viven y a la vez en las que participan, más que ser reconocidos desde la política como "juventud”. De esto se desprende que la condición juvenil es una construcción sociocultural, definida históricamente, cambiante y transitoria, que se presenta como parte de los procesos de disputa y negociación, entre las representaciones externas y las propias de los sujetos jóvenes en esta condición; lo anterior teniendo en cuenta la heterogeneidad cultural y la desigualdad estructural que perfilan la condición juvenil y las identificaciones laborales específicas.

Es evidente que desde nuestros espacios académicos y partiendo desde la vida cotidiana de los jóvenes para explorar sus experiencias, nos impulsa a repensar que si se quiere generar una alternativa a las posturas hegemónicas, hay que generar desde los escenarios donde vive, trabaja e interactúa la gente, un pensamiento y unas ciencias sociales críticas capaces de revertirlas, de generar otros mundos posibles, que tenga en cuenta también la relación capital-trabajo en un contexto de mayor equidad en especial con la masa de trabajadores informales, jóvenes flexibles y que permita la afiliación social de los desafiliados que habitan las ciudades y zonas rurales, sin esperanza de formar parte de la sociedad de la que han sido expulsados. 


\section{Notas}

${ }^{1}$ El artículo de reflexión se constituye en uno de los insumos teóricos de la investigación doctoral que se realiza en la Universidad de Manizales- CINDE, sobre las narrativas de identidad desde la experiencia de trabajo en sujetos jóvenes: el caso de los vendedores callejeros.

2 Por "gubernamentalidad" entiendo el conjunto constituido por las instituciones, los procedimientos, análisis y reflexiones, los cálculos y las tácticas que permiten ejercer esta forma específica, tan compleja, de poder, que tiene como meta principal la población, como forma primordial de saber, la economía política, como instrumento técnico esencial, los dispositivos de seguridad” (Foucault 1999:195). 


\section{Bibliografía}

Acebedo, L. (2010), Manizales: de Eje del conocimiento a "Call Center", Universidad de Manizales.

Alpízar, L, Bernal M. (2003), “La construcción social de las juventudes”, en Última década, 19, Santiago

Alonso, L.E (1999), Trabajo y ciudadanía. Estudios sobre la crisis de la sociedad salarial, Trotta, Fundación 1o de Mayo, Madrid

Aravena, A. (2001), Los estudios del trabajo en América latina: apuntes para el debate, Disponible en http://www.estudiosdeltrabajo.cl/wp-content/ uploads/2008/11/los-estud1.doc

Battistini O. (Comp.), (2004), El trabajo frente al espejo. Continuidades y rupturas en los procesos de construcción identitaria de los trabajadores, Prometeo, Buenos Aires

Bauman, Z. (2001), “En Busca de agencia”, en En Busca de la Política, Fondo de Cultura Económica, México. D.F.

Ídem (2007), Tiempos líquidos. Vivir en una época de incertidumbre, Tusquets Editores, Barcelona

Ídem (2006), “Ley global, ordenes locales” en La Globalización, consecuencias humanas. Fondo de Cultura Económica, México.

Beck, U. (1998), "Errores del globalismo. Respuestas a la globalización. Partes tercera y cuarta”, en ¿Qué es la globalización?: falacias del globalismo, respuestas a la globalización, Paidós, Barcelona.

Bialakowsky, A, Costa, M.I, Patrouilleau, M. (2009), “Aportes a una teoría del cambio. Gubernamentalidad, fuerzas productivas y praxis de sujetos colectivos en nueva época”, en Neffa JC, De la Garza, E, Muñiz, L (Comp.), Trabajo, empleo, calificaciones profesionales, relaciones de trabajo e identidades laborales, Consejo Latinoamericano de Ciencias Sociales, CLACSOCAICyT, Buenos Aires.

Carpio, J, Klein, E, Novacovsky, I. (Comps.) (2000), Informalidad y exclusión social, Fondo de Cultura Económica-Organización Internacional del Trabajo, Buenos Aires.

Castel, R. (2004), La metamorfosis de la cuestión social. Una crónica del salariado, Paidós, Buenos Aires.

Ídem (2010), El ascenso de las incertidumbres. Trabajo, protecciones, estatuto del individuo, Fondo de Cultura Económica, Buenos Aires 
Castellanos, J.M. (2011), “La condición juvenil: opciones metodológicas para la construcción de un objeto de conocimiento”, en Muñoz G. (Ed), Jóvenes, culturas y poderes, Siglo del Hombre Editores, Bogotá

Cortés, F. (2000), "La metamorfosis de los marginales; la polémica sobre el sector informal en América Latina”, en De la Garza E (Coord.), Tratado latinoamericano de sociología del trabajo, El Colegio de México, UAM, Fondo de Cultura Económica, México.

De la Garza, E. (2009), “Hacia un concepto ampliado del trabajo, de control, de regulación y de construcción social de la ocupación: los otros trabajos” en Revista Iztapalapa No. 66, año 30, UAM-I, México.

Ídem (2001), "El papel del concepto trabajo en la teoría social del siglo XX”, en Tratado latinoamericano de sociología del trabajo, El Colegio de México, Universidad Autónoma Metropolitana, Fondo de Cultura Económica, México.

Ídem (1994), “Las teorías de la elección racional y el marxismo analítico”, en Estudios Sociológicos, vol. XII, No. 35, El Colegio de México, México.

Delfin, M., Pichetti, V. (2004), “De la fábrica al barrio y del barrio a las calles. Desempleo y construcción de identidades en los sectores populares desocupados del conurbano bonaerense”, en Battistini O. (Comp.), El trabajo frente al espejo. Continuidades y rupturas en los procesos de construcción identitaria de los trabajadores, Prometeo, Buenos Aires.

Feixa, C., Leccardi, C. (2011), “El concepto de generación en las teorías sobre la juventud”, en Muñoz G. (Ed), Jóvenes, culturas y poderes, Siglo del Hombre Editores, Bogotá.

Foucault, M. (1999), “La gubernamentalidad”, en Estética, Ética y Hermenéutica. Obras Esenciales: Vol III, Paidós Básica, Barcelona.

García Canclini, N. (1989), “Capitales de la cultura y ciudades globales y Hacia una agenda cultural de la globalización”, en La globalización imaginada. Paidós, Argentina.

García Canclini, N, Cruces, F, Urteaga, M. (2012), Redes, culturas urbanas y redes digitales, Ariel. Colección Fundación Telefónica, Barcelona.

Gorz, A. (2000), Miserias del presente, riquezas de lo posible, Paidós, Buenos Aires.

Hopenhayn, M., Morán, M. (2008), “Inclusión y ciudadanía. Perspectivas de la juventud en Iberoamérica”, en Pensamiento Latinoamericano, Fontana y Gillsans, Madrid. 
Hopenhayn, M. (2001), Repensar el trabajo. Historia, profusión y perspectivas de un concepto, Norma, Buenos Aires.

Margullis, M. (2001), “Juventud: una aproximación conceptual”, en Solum D. (Comp), Adolescencia y juventud en América Latina, Libro Universitario Regional, Cartago.

Margullis, M., Urresti, M. (1998), “La construcción social de la condición de juventud”, en Viviendo a toda. Jóvenes, territorios culturales y nuevas sensibilidades, Universidad Central, Siglo del Hombre Editores, Bogotá.

Martin, M. (2008), Juventud, educación y trabajo. La dinámica entre estructuras y agentes burocráticos en las políticas de Mendoza, Tesis de Doctorado en Ciencias Políticas y Sociales, Universidad Nacional de Cuyo, Mendoza.

Muñoz, G. (2011), “La relación de los jóvenes y las jóvenes con la cultura y el poder”, en Muñoz G. (Ed), Jóvenes, culturas y poderes, Siglo del Hombre Editores, Bogotá.

Ídem (2011), “Practicas políticas de jóvenes: desde abajo y a la izquierda”, en Amador J.C, García R y Leonel Q.M. (Ed), Jóvenes y derechos en la acción colectiva: voces y experiencias de organizaciones juveniles en Bogotá, Universidad Distrital Francisco José de Caldas-Personería de Bogotá, Bogotá

Neffa, J.C. (2001), "Presentación del debate reciente sobre el fin del trabajo”, en Neffa J.C y De la Garza, E. (Comp.), El trabajo del futuro. El futuro del trabajo, Consejo Latinoamericano de Ciencias Sociales, CLACSO, Grupo de Trabajo: Trabajo, sujetos y organizaciones laborales, Buenos Aires.

Offe, C. (1996), “Pleno empleo, ¿una cuestión mal planteada?”, en Sociedad $\mathrm{N}^{\circ}$ 9, Facultad de Ciencias Sociales, Universidad de Buenos Aires, Buenos Aires, Disponible en http://www.primerolagente.com.ar/ modelobm.htm

Ortiz, R. (1998), Otro territorio, Convenio Andrés Bello, Santafé de Bogotá.

Pérez Islas, J. (2010), Nueva crisis ¿¿una reestructuración de la condición juvenil? Construyendo hipótesis, en Curso Jóvenes, educación y trabajo. Nuevas tendencias y desafíos, FLACSO-Argentina.

Ídem (2008), “Juventud un concepto en disputa”, en Teorías sobre la juventud. Las miradas de los clásicos, Universidad Nacional Autónoma de México, México.

Piña Mendoza, C. (2004), Cuerpos posibles... cuerpos modificados. Tatuajes y perforaciones en jóvenes urbanos, SEP/IMJ-CIEJ, México. 
Reguillo, R. (2010), “La condición juvenil en el México contemporáneo. Biografías, incertidumbre y lugares”, en Reguillo, Rossana (Coord.), Los jóvenes en México, Fondo de Cultura Económica. Consejo Nacional para la Cultura y las Artes, México

Ídem (2000), Emergencia de culturas juveniles. Estrategias del desencanto, Norma, Bogotá.

Rifkin, J. (1996), El fin del trabajo. Nuevas tecnologías contra puestos de trabajo: el nacimiento de una nueva era, Paidós Ibérica S.A., Barcelona.

Rossi, F. (2006), Documento No. 1: Las juventudes en movimiento: Informe sobre las formas de participación política de los jóvenes, s.e, Buenos Aires.

Rosanvallon, P. (1995), La nueva cuestión social, Manantial, Buenos Aires.

Sen, A. (2002), "La pobreza como privación de capacidades y Mercados, Estado y oportunidad social”, en Desarrollo y libertad, Planeta, Barcelona.

Stiglitz, J.E. (2006), “La carga de la deuda”. “Reformar el sistema global de reservas. Democratizar la globalización”. Notas”, en: Cómo hacer que funcione la globalización. Colombia: Taurus.

Valenzuela, J.M. (2005), “Decálogo para repensar las certezas”, en El futuro ya fue, s.e, México.

Recibido: 05.05.2013

Aceptado: 13.08.2014 